\title{
Superior Oblique Myokymia Presumed Due to Large Posterior Fossa Arteriovenous Malformation
}

\author{
Laura Donaldson (D, Brian van Adel, Amadeo R. Rodriguez,
}

Keywords: Diplopia, Superior oblique myokymia, Arteriovenous malformation

doi:10.1017/cjn.2020.125

Can J Neurol Sci. 2020; 47: 824-825

A 26-year-old female presented with a complaint of intermittent oscillopsia and binocular vertical diplopia for the past 5 years. Over the past several months, she had noticed intermittent pulsatile tinnitus. She was otherwise healthy with no previous history of trauma and had no other visual or neurologic complaints. In Neuro-ophthalmology clinic, she was found to have 20/15 vision in both eyes with full ocular motility. There was a small exophoria in primary position and small esophoria in downgaze. Her slit lamp and fundus examinations were normal. During the assessment, the left eye was noted to undergo highfrequency, small amplitude incyclotorsional oscillations for a few seconds at a time (Video 1 in the supplementary material), which she was able to provoke by looking down. The diagnosis of superior oblique myokymia was made, and an MRI/MRA of the brain was requested.

MRI of the brain revealed a large cerebellar arteriovenous malformation (AVM) (Figure 1A, 1B). The nidus was located within the left cerebellar hemisphere, with feeding vessels appearing to arise mainly from the left vertebrobasilar system. Multiple interconnected vascular loops were present within the cerebellar hemispheres. Dilated, tortuous draining veins were present in the interpeduncular, ambient and prepontine cisterns, including vessels wrapping around the cerebral peduncles in the expected course of the trochlear nerve.

The patient underwent a diagnostic cerebral angiogram (Figure 1C, 1D), which showed a large compact AVM nidus measuring $6 \mathrm{~cm}$ in diameter within the left cerebellar hemisphere. Arterial supply was from the left and right posterior inferior cerebellar arteries, left anterior inferior cerebellar artery and left superior cerebellar artery. Multiple ecstatic and dilated veins drained the AVM to both transverse sinuses as well as reflux into the deep venous system via the left basal vein of Rosenthal. Multiple high-flow arteriovenous (AV) shunts were noted predominately within the left cerebellar hemisphere with multiple dilated, and tortuous cerebellar hemispheric draining veins with a pseudophlebitic pattern of the pial veins of the posterior cranial fossa suggestive of long-standing AV shunting and venous hypertension.

For symptomatic treatment, the patient was started on topical timolol $0.5 \%$ bid to the left eye, an off-label use. She reported a noticeable decrease in the frequency of her oscillopsia. Later, endovascular embolization of the AVM (Figure 1E-1H) was performed, resulting in significant improvement and allowing her to discontinue timolol postoperatively.

Superior oblique myokymia is a monocular condition that can occur in association with any structural compromise to the trochlear nerve including microvascular compression, ${ }^{1,2}$ trauma and brainstem neoplasms, though in many cases no underlying etiology can be identified. The reported success of timolol in treating this condition could relate to a reduction in ephatic transmission or decreased amplitude of vascular pulsations through blood pressure lowering. ${ }^{3}$ As in this case, definitive treatment consists of decompression of the affected trochlear nerve.

It has been suggested that neuroimaging may not be required for every case of superior oblique myokymia, but should be considered when there is non-response to topical therapy and certainly before planning for extra-ocular muscle surgery. ${ }^{4}$ In this case, it was the long-standing nature of her complaint and relatively severe presentation with frequent, bothersome episodes which prompted early referral for MRI.

\section{CONFLict of InTERest}

None.

\section{STATEMENT OF AUTHORSHIP}

LD wrote the manuscript; BV and ARR made revisions and approved the final version.

\section{SUPPLEMENTARY MATERIAL}

To view supplementary material for this article, please visit https://doi.org/10.1017/cjn.2020.125.

From the Department of Surgery, Division of Ophthalmology, Hamilton Regional Eye Institute, McMaster University, Hamilton, Ontario, Canada (LD, ARR); Division of Neurology, Neurosurgery, and Diagnostic Imaging, Hamilton General Hospital, McMaster University, Hamilton, Ontario, Canada (BA); and Department of Medicine, Division of Neurology, McMaster University, Hamilton, Ontario, Canada (ARR)

Received December 21, 2019. Final Revisions Submitted April 22, 2020. Date of Acceptance June 9, 2020.

Correspondence to: Amadeo Rodriguez, Hamilton Regional Eye Institute, 2757 King St E, Hamilton, ON L8G 4X3, Canada. Email: arodrig@mcmaster.ca 

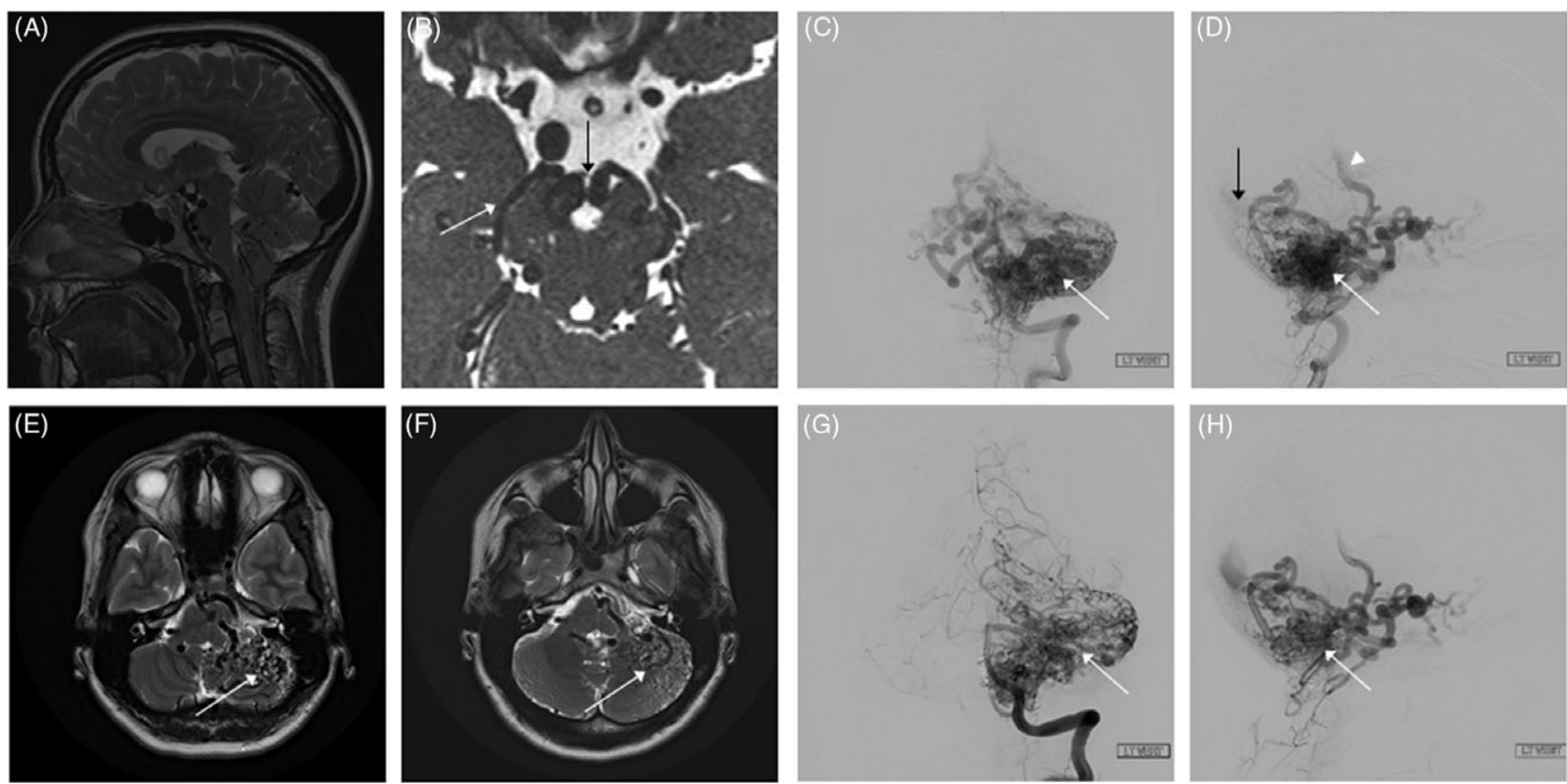

Figure 1: (A) Sagittal T2 and (B) axial fast imaging employing steady-state acquisition magnetic resonance images showing multiple, abnormal dilated vessels in the cerebellum and brainstem. Tortuous vessels are present surrounding the midbrain in the interpeduncular (black arrow) and ambient cistern (white arrow) along the course of the trochlear nerve. $(C, D),(G, H)$ : Digital subtraction angiography of the cerebral vessels. $(C, D) A-P(C)$ and lateral $(D)$ pre-embolization views showing filling from the left vertebral artery. A large cerebellar arteriovenous malformation (AVM) with a compact nidus in the left cerebellar hemisphere (white arrow) is present with complex venous drainage to the transverse sinuses (black arrow) and deep cerebral veins (white arrowhead). (E, F) Pre- (E) and post- $(F)$ embolization MRI axial T2 sequences at the level of the pons and cerebellum. MRI 12 months post embolization shows a reduction in the size of the AVM nidus (white arrow) and less prominent draining veins. $(G, H)$ Post-embolization (Onyx) anteroposterior $(G)$ and lateral $(H)$ angiograms of the AVM after selective injection of the left vertebral artery demonstrate a reduction in the overall size of the AVM nidus (white arrows) and the degree of AV shunting.

\section{REFERENCES}

1. Fam MD, Scott C, Forster A, Kamel MH. Microvascular decompression for superior oblique myokymia: case report. Br J Neurosurg. 2014; 28(4):552-5.

2. Hashimoto M, Ohtsuka K, Suzuki Y, Minamida Y, Houkin K. Superior oblique myokymia caused by vascular compression. J Neuroophthalmol. 2004;24(3):237-9.
3. Strupp M, Dieterich M, Brandt T, Feil K. Therapy of vestibular paroxysmia, superior oblique myokymia, and ocular neuromyotonia. Curr Treat Options Neurol. 2016;18(7):34.

4. Zhang M, Gilbert A, Hunter DG. Superior oblique myokymia. Surv Ophthalmol. 2018;63(4):507-17. 